\title{
Population synthesis of planetary nebulae from binaries
}

\author{
Maxwell $\mathrm{Moe}^{1}$ and Orsola De Marco ${ }^{2}$ \\ ${ }^{1}$ Harvard-Smithsonian Center for Astrophysics \\ 60 Garden Street, Cambridge, MA, 02138, USA \\ email: mmoe@cfa.harvard.edu \\ ${ }^{2}$ Research Centre for Astronomy, Astrophysics \& Astrophotonics and \\ Department of Physics \& Astronomy, Macquarie University, Sydney, NSW 2109, Australia
}

\begin{abstract}
There is at present no viable theory whereby a single star with $M_{\mathrm{MS}}<2.3 \pm 0.3 \mathrm{M}_{\odot}$ can sustain a PN-forming superwind with $\dot{M}>3 \times 10^{-5} \mathrm{M}_{\odot} \mathrm{yr}^{-1}$ at the tip of the AGB that can eject the last $\sim 0.3 \mathrm{M}_{\odot}$ of envelope mass. We propose that a binary companion can not only result in non-spherical $\mathrm{PN}$, but more importantly provide the AGB mass loss rate enhancement that is required to create a visible PN. We provide an overview of our binary population synthesis calculations of the PN formation rates from common envelope (CE) interactions, CE mergers with substellar companions, tidally synchronized systems that avoid CE, gravitationally focused winds, and double degenerate systems. The predicted number of Galactic PN with radii < $0.9 \mathrm{pc}$ shaped and created by a binary companion is $8,100 \pm 2,300$ which is $(71 \pm 20) \%$ of the observationally-estimated total. We demonstrate that the observed close central star of PN binary fraction of $15-20 \%$ is consistent with our overall binary fraction, considering we predict two binary populations with period distributions centred at $\log \mathrm{P}($ days $) \sim 0$ and 4 . Finally, we discuss the impact of binarity on the PN luminosity function, central star mass distribution, chemical abundances, morphologies, etc., and why these distributions predicted in the binary scenario are close to observations while the single star paradigm produces distributions which are measurably discrepant.
\end{abstract}

Keywords. planetary nebulae: general, binaries: general, stars: AGB and post-AGB

\section{Introduction}

Recent dynamical modeling has demonstrated the difficulty in maintaining the necessary rotation rate and/or magnetic dynamo in single asymptotic giant branch (AGB) stars to shape the subsequent planetary nebula (PN; Nordhaus et al. 2007). Although shaping by a companion is certainly a viable avenue in creating the diverse PN morphologies, the observed fraction of close binary CSPN is rather small at $\sim 15-20 \%$ with an indication of a turn-over in periods beyond $\sim 1$ day (Miszalski et al. 2009). Moreover, if one supposes that non-spherical PN derive from interactions with a stellar or substellar companion, then the immem diate conclusion is that most single stars do not produce a visible $\mathrm{PN}$ because the fraction of stars with companions having the necessary mass ratios and periods to shape the AGB star is only a small subset of all available progenitors (for a review, see De Marco 2009).

In Moe \& De Marco (2006, hereafter Paper I), we used a population synthesis technique to predict a current Galactic number of $\sim 50,000 \mathrm{PN}$ with $r<0.9$ pc, assuming all progenitors with $M_{\mathrm{MS}} \gtrsim 0.9 \mathrm{M}_{\odot}$ produced a visible $\mathrm{PN}$. This result is $\sim 5$ times higher than the observational estimate inferred from either local PN counts $(\sim 12,000 \mathrm{PN}-$ Frew \& Parker 2007) or extragalactic PN rates ( 10,000 PN - Peimbert 1990) after scaling to our PN radius requirement. This suggests that only $\sim 20 \%$ of WDs evolve through a PN 
phase. Our goal is to provide a self-consistent paradigm to determine which AGB stars produce visible $\mathrm{PN}$.

\section{Population Synthesis}

The superwind paradigm. In order to calculate which progenitors are capable of producing a PN, we rely on the AGB and PN observations themselves combined with the interacting winds theory (Kwok et al. 2000):

$$
M_{\mathrm{PN}}=\dot{M}_{\mathrm{AGB}}\left(\frac{v_{\mathrm{PN}}}{v_{\mathrm{AGB}}}-1\right) \tau_{\mathrm{PN}}
$$

where the typical $\mathrm{PN}$ with age $\tau_{\mathrm{PN}} \sim 10,000$ yrs has nebular mass $M_{\mathrm{PN}} \sim 0.2 \mathrm{M}_{\odot}$ and mean $\mathrm{PN}$ expansion velocity $\mathrm{v}_{\mathrm{PN}} \sim 28 \mathrm{~km} \mathrm{~s}^{-1}$, and we implement the wind velocity at the tip of the AGB to be $\mathrm{v}_{\mathrm{AGB}} \sim 18 \mathrm{~km} \mathrm{~s}^{-1}$ (Ramstedt et al. 2009). This implies that in order to produce a PN, an AGB star must sustain a superwind $\dot{M}_{\mathrm{AGB}} \gtrsim 3 \times 10^{-5}$ $\mathrm{M}_{\odot} \mathrm{yr}^{-1}$ during the time when the envelope mass diminishes from $\mathrm{M}_{\text {env }} \sim 0.3 \mathrm{M}_{\odot}$ to $10^{-4}-10^{-3} \mathrm{M}_{\odot}$. We assume that AGB progenitors that fail to meet this criterion will evolve too slowly across the post-AGB track, thereby leaving a "naked" central star with a PN that is too dispersed to be observed (Soker \& Subag 2005).

Applying various AGB mass loss prescriptions to the PN-formation definition of a superwind (not the canonical definition), one finds a large range of limiting progenitor masses $\mathrm{M}_{\mathrm{min}}=1.3-2.6 \mathrm{M}_{\odot}$ below which a single star cannot produce a PN (Wachter et al. 2002, Marigo \& Girardi 2007, Willson priv. comm.). We present here our adopted value of $\mathrm{M}_{\min }=2.3 \pm 0.3 \mathrm{M}_{\odot}$ which is constrained by population synthesis to make the predicted numbers of PN with $r<0.9 \mathrm{pc}$ match the observations (see below). Albeit high, it is conceivable, given the uncertainty in AGB mass loss evolution, that single stars with main sequence mass as high as this limit will not make bright, young PN.

$P N$ formation from binaries. Low mass progenitors can still produce a PN if they sustain a strong binary interaction that can enhance the mass loss rate on the AGB. This can be achieved in the form of a common envelope (CE) with a stellar or WD companion in which the companion in-spirals significantly but emerges at periods of a few hours to days (Paczynski 1976). In addition, increasing the rotation rate of the AGB star to $\sim 15 \%$ of its Keplerian value can enhance the mass loss rate by the necessary factor of $\sim 2-3$ (Dorfi \& Hoefner 1996). Increased rotation may be attained via a CE merger with a brown dwarf or tidal synchronization with a stellar companion that avoids CE. In addition, more massive progenitors that will achieve a superwind by their own merits can still be shaped via a weak binary interaction such as a merger with a planet or gravitational focusing of the winds by companions in even wider orbits.

We employ recent stellar models combined with different prescriptions for tides, CE evolution, etc. to determine which binaries evolve through the various PN formation channels. We then use our results from Paper I alongside the observed binary fraction, mass-ratio distribution, and period distribution to infer the birthrates of PN that derive from weak and strong binary interactions. We assume PN with $r<0.9$ pc have a visibility time of $\sim 32,000$ yrs minus the time it takes the CS to evolve to ionizing temperatures, but take into account that post-CE binaries will have accelerated CS evolutions, while post-AGB binaries with periods $~ 100-2,000$ days that form a disk will have stalled postAGB evolutions and be incapable of producing PN (van Winckel 2003). We point out here that because the ratio of maximum AGB to RGB radii increases with increasing mass, more massive progenitors are more likely to evolve via a binary interaction on the AGB. 


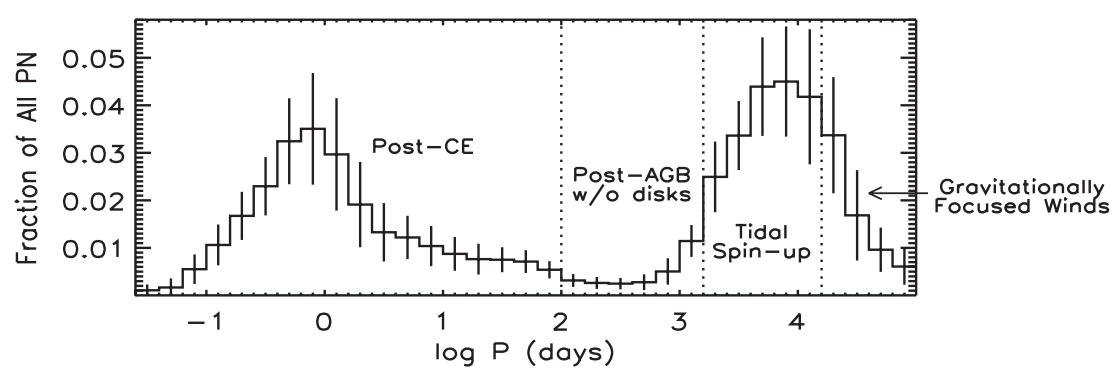

Figure 1. Predicted period distribution of PN that derive from weak and strong binary interactions on the AGB. We expect only $\sim 25 \%$ of $\mathrm{PN}$ to derive from post-CE binaries while the binaries that avoid $\mathrm{CE}$ are left at longer periods such as those that were tidally synchronized $(\sim 15 \%)$ and those that induced gravitationally focused winds $(\sim 10 \%)$. We also predict that $\sim 20 \%$ of PN derive from CE mergers with brown dwarfs and planets, leaving only $\sim 30 \%$ of PN that can derive from more massive single stars and wide binaries in order for the predicted numbers of PN in the Galaxy to match the observations. We note that we have excluded post-AGB binaries with disks considering they are unlikely to evolve into PN, as is indicated by the dip at periods $\sim 100-2,000$ days.

\section{Results and Trends}

We predict a total of $8,100 \pm 2,300 \mathrm{PN}$ with $r<0.9 \mathrm{pc}$ in the Galaxy that derive from binaries that have undergone either strong interactions on the AGB, or weak interactions on the AGB with primaries $M_{\mathrm{MS}}>2.3 \pm 0.3 \mathrm{M}_{\odot}$. Comparing with the observationallyestimated total, we infer that $(71 \pm 20) \%$ of PN with $r<0.9$ pc derive from binary interactions while the remaining $\sim 30 \%(3,300 \pm 2,700)$ derive from single stars and wide binaries. Integrating down the single star progenitor mass distribution from Paper I, we find this single star PN number corresponds to a limiting mass of $M_{\mathrm{MS}}=2.3 \pm 0.3 \mathrm{M}_{\odot}$ below which a single star cannot make a PN with $\mathrm{r}<0.9 \mathrm{pc}$, which is how we constrained $\mathrm{M}_{\text {min }}$. We note that the PN binary fraction of $\sim 70 \%$ derived above is for the entire Galaxy, but we calculate that it is $(89 \pm 11) \%$ for the bulge, where there are fewer single star progenitors with $M_{\mathrm{MS}}>\mathrm{M}_{\mathrm{min}}$. We emphasize that these statistics should only be applied to PN with $r<0.9$ pc. Larger PN with ages $\gtrsim 32,000$ yrs would have allowed the CS of single star progenitors with $M_{\mathrm{MS}}<\mathrm{M}_{\text {min }}$ to transition to hot enough temperatures to photoionize the nebula, indicating the single star fraction will be higher for older PN.

In Fig. 1, we display our predicted period distribution of $\mathrm{PN}$ that have evolved through strong or weak binary interactions. We demonstrate that the observed $\sim 15-20 \%$ close binary fraction (Miszalski et al. 2009) is indicative of a much higher binary fraction, even if the distribution turns over beyond periods $P \sim 1$ day.

Using the dynamical age and location on the HRD to constrain the CS properties, observations indicate the CS mass distribution peaks near $\sim 0.61 \mathrm{M}_{\odot}$ (Gesicki \& Zijlstra 2007), which is inconsistent with our predicted peak near $\sim 0.57 \mathrm{M}_{\odot}$ assuming the canonical single star paradigm (see Paper I). However, taking into account that binaries with more massive primaries will be more likely to undergo an interaction on the AGB, the CS mass distribution is shifted toward higher values. Even more important, the measured CS masses are based on the evolutionary tracks of single stars, and post-CE systems will have accelerated post-AGB evolution which may cause the CS mass to be overestimated. Considering these selection and biasing effects, we are able to reproduce the observed CSPN mass distribution, but only if low mass single stars do not produce PN and $\sim 25 \%$ of $\mathrm{PN}$ derive from post-CE binaries.

It has been suggested that the constancy of the bright end of the PN luminosity function (PNLF) is due to blue stragglers or contamination by symbiotic binaries (Ciardullo 
2010, references therein). We propose a third option whereby the metallicity dependent initial-to-final mass relation which leads to higher core masses in lower metallicity systems can compensate for the fact that older, lower metallicity populations will have lower mass progenitors now evolving into PN. We also point out the difficulty in reconciling the normalization of the PNLF in older populations such as ellipticals, and our population synthesis suggests that in these older systems the visibility time is the typical $\sim 10,000$ yrs for PN near the bright end, but that the fraction of WDs that pass through a PN phase is even lower at $\sim 5-10 \%$ compared to the Galaxy at $\sim 20 \%$.

One may suspect that because a binary interaction will curtail the AGB evolution, that the expected chemical abundances of PN formed by binaries will automatically be shifted toward chemistries with less carbon, s-process elements, and nitrogen (for more massive stars). However, the opposite can occur when the dominant effect is not when a binary companion interacts with the AGB primary, but which binaries will interact and produce PN. As mentioned, because the ratio of the AGB radius to RGB radius increases with increasing mass, the binary formation channel of PN is skewed toward higher masses, and therefore more chemically enriched PN. After implementing these two competing effects in our population synthesis, we can better reproduce the observed dual-dust chemistries in bulge PN (Górny et al. 2010). Also, the observed fraction of Type I PN that are suspected to derive from progenitors $M_{\mathrm{MS}} \gtrsim 4.0 \mathrm{M}_{\odot}$ is $\sim 21 \%$, which is inconsistent with the single star paradigm prediction of $(5 \pm 3) \%$ (see Paper I, references therein), but consistent with our superwind paradigm (binaries plus massive single stars) prediction of $(17 \pm 5) \%$.

The predicted fraction of $\sim 70 \%$ of $\mathrm{PN}$ that derive from binaries corresponds well to the fraction of PN with nonspherical morphologies (Soker 1997). Although there is a higher fraction of spherical PN at higher galactic latitudes implying lower mass progenitors, we maintain this is a selection effect whereby the brighter bipolar and elliptical PN that are further away biases the ratio in the plane. Finally, because $M_{m i n}$ can be lower for older PN, we expect there to be an even higher fraction of larger, spherical PN r $>0.9 \mathrm{pc}$ deriving from single stars $M_{\mathrm{MS}} \sim 1.5-2.3 \mathrm{M}_{\odot}$.

MM acknowledges support by the National Science Foundation (NSF) Graduate Research Fellowship Program under Grant \#'s DGE-0644491 and DGE-0946799.

\section{References}

Ciardullo, R., 2010, PASA, 27, 149

De Marco, O., 2009, PASP, 121, 316

Dorfi, E. A. \& Hoefner, S., 1996, A\&A, 313, 605

Frew, D. J. \& Parker, Q. A., 2007, APN IV, 68

Gesicki, K. \& Zijlstra, A. A., $A \& A, 467,29$

Górny, S. K., et al., 2010, $A \mathscr{E} A$, 516, 39

Kwok, S., 2000, Camb. Astrophys. Ser., Vol. 33

Marigo, P. \& Girardi, L., 2007, A\&SA, 469, 239

Miszalski, B., Acker, A., Moffat, A. F. J., Parker, Q. A., \& Udalski, A., 2009, A\&A, 496, 813

Moe, M. \& De Marco, O., 2006, ApJ, 650, 916, Paper I

Nordhaus, J., Blackman, E. G., \& Frank, A., 2007, MNRAS, 376, 599

Paczynski, B., 1976, IAU Symposium 73, 75

Peimbert, M., 1990, RMxAA, 20, 119

Ramstedt, S., Schöier, F. L., \& Olofsson, H., 2009, 499, 515

Soker, N., 1997, ApJS, 112, 487

Soker, N. \& Subag, E., 2005, AJ, 130, 2717

van Winckel, H., 2003, ARA $\& A$, 41, 391

Wachter, A., et al., 2002, A\&\&A, 384, 452 